\title{
Cooperative Robot Assistant for Retinal Microsurgery
}

\author{
Ioana Fleming ${ }^{1, *}$, Marcin Balicki ${ }^{1}$, John $\mathrm{Koo}^{2}$, Iulian Iordachita ${ }^{1}$, Ben Mitchell ${ }^{1}$, \\ James Handa ${ }^{2}$, Gregory Hager ${ }^{1}$, and Russell Taylor ${ }^{1}$ \\ ${ }^{1}$ ERC for Computer Integrated Surgery, Johns Hopkins University \\ ${ }^{2}$ Wilmer Eye Institute, Johns Hopkins Medical, Baltimore, MD, USA \\ \{inicola1, marcin, iordachita, rht\}ajhu.edu
}

\begin{abstract}
This paper describes the development and results of initial testing of a cooperative robot assistant for retinal microsurgery. In the cooperative control paradigm, the surgeon and the robot share control of a tool attached to the robot through a force sensor. The system senses forces exerted by the operator on the tool and uses this information in various control modes to provide smooth, tremor-free, precise positional control and force scaling. The robot manipulator is specifically designed with retinal microsurgery in mind, having high efficacy, flexibility and ergonomics while meeting the accuracy and safety requirements of microsurgery. We have tested this robot on a biological model and we report the results for reliably cannulating $\sim 80 \mu \mathrm{m}$ diameter veins (equivalent in size to human retinal veins). We also describe improvements to the robot and the experimental setup facilitating more advanced set of experiments.
\end{abstract}

Keywords: cooperative robot control, retinal microsurgery, vein cannulation.

\section{Introduction}

Retinal disease remains the leading cause of new blindness in the Western World. The World Health Organization estimates that "over 180 million people are visually disabled world wide and that 40 to 45 million of these are blind" [1]. In the current ophthalmologic practice several pathologies receive indications for vitreoretinal surgery, typically in the elderly: retinal detachment, vitreous hemorrhage, macular pucker, macular hole and diabetic retinopathy. The main challenge linked to microsurgical procedures is to be able to perform surgical gestures on structures with cross sections varying from millimeters down to microns. With respect to retinal microsurgery, the manipulation of vitreoretinal structures is particularly difficult given their relative delicacy, inability to regenerate if injured, surgical inaccessibility, and lack of sensory feedback using today's manual tools and direct optical visualization. The cooperative robotic assistant presented here addresses a number of these restrictions. For the initial evaluation of our robot system, we have chosen retinal vein cannulation as a target application that exhibits these limitations.

The conventional vitreoretinal surgical system uses an operating microscope to visualize surgical instruments that are placed in three 20-25 gauge diameter sclerotomy incisions. Surgical tasks require delicate maneuvers which are physically not

* Supported in part by the National Science Foundation under ERC grant EEC9731748. 
possible for many ophthalmologists due to inability to visualize small structures, tremor or insufficient dexterity. Manual dexterity, precision and perception are particularly important during tasks where the ability to position instruments with great accuracy correlates directly with successful outcome [2], [3].

While it is possible to position an instrument briefly at an intended target with great accuracy, maintaining this position for extended periods of time becomes increasingly difficult due to physical, visual and mental fatigue. Retinal vein cannulation requires the insertion of a needle into a vein on the surface of the retina as a path for drug delivery. Specifically for this procedure, a principal barrier to wider development and testing is the extreme difficulty of not only cannulating blood vessels but also maintaining the cannulation for several seconds. Physiological tremor, reportedly $108 \mu \mathrm{m}$ in amplitude [4], is generally greater than the target blood vessel diameter on the order of $80 \mu \mathrm{m}$.

There is extensive literature reporting robotic systems for surgery (e.g., [5]), including commercially deployed systems (e.g., [6]). A number of researchers have proposed master-slave microsurgical systems (e.g., [7]), including some systems for the eye ([8]). With the exception of exploratory work by Hunter et al. [9], most of this work has focused on direct improvement of a surgeon's ability to manipulate tissue remotely or at a very fine scale, rather than exploiting the ability of the computer to assist the surgeon more broadly.

In contrast, the JHU Steady-Hand Robot (SHR) [10], [11] was designed to cooperatively share control of a surgical tool with the surgeon while meeting the performance, accuracy, and safety requirements of microsurgery, however it was not particularly suitable for vitreoretinal surgery. Some of this system's limitations were addressed in the design of our current retinal microsurgery robot which is more ergonomically convenient for the surgeon and is complemented by $3 \mathrm{D}$ visualization software [12]. This paper reviews system design, followed by the experimental setup and results of $\sim 80 \mu \mathrm{m}$ OD vein cannulation task in a biological model, and a new framework for retinal surgery.

\section{System Design}

The design of the robot prototype began with an analysis of the necessary degrees of freedom (DOF), options for obtaining a remote center of motion (RCM), and establishment of specifications for mechanical parameters such as range of motion, precision, and maximum velocity [12], [13]. The final prototype was controlled using MEI servo motor controllers, while high level admittance controller was implemented in software $(\mathrm{C}++)$ and operated in Windows XP envi-

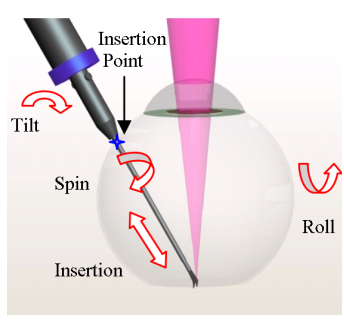

Fig. 1. DOF for retinal surgery tool ronment on an industrial grade desktop computer.

In establishing the specifications (Table 1) and the required degrees of freedom (Fig. 1) for the robot mechanical system, we considered its interaction with patient 
Table 1. Robot performance specifications for approach phase (A), insertion phase (I), and retinal surgery phase $(\mathrm{R})$ motions

\begin{tabular}{llll}
\hline Robot Specification & Units & Value \\
\hline Roll/tilt motion & & degrees & \pm 30 \\
XYZ motion & $\mathrm{mm}$ & \pm 50 \\
\hline Roll/tilt precision & radians & $\sim 0.00005$ \\
XYZ precision & $\mu \mathrm{m}$ & $\sim 2$ \\
Net precision at retina & & $\mu \mathrm{m}$ & $\sim 5$ \\
\hline Cartesian tip speed & - phase A & $\mathrm{mm} / \mathrm{s}$ & 10 \\
& - phase I & $\mathrm{mm} / \mathrm{s}$ & 5 \\
& - phase $\mathrm{R}$ & $\mathrm{mm} / \mathrm{s}$ & $<1$ \\
\hline
\end{tabular}

anatomical structures, surgeon workspace, and imaging system. Other important factors were the patient safety in correlation with surgery accuracy.

The robot mechanical system consists of three major parts (Fig. 2A): the XYZ system, the roll mechanism, and the tilt mechanism. The XYZ system assures the global motions of the surgical tool. The roll mechanism, consisting of a rotating table, was tilted at $-15^{\circ}$ from the horizontal direction to assure better access of the surgical tool to the eye depression of the patient face. This roll mechanism configuration is appropriate for the actual tilt mechanism type and for a robot located on the same side of the face as the targeted eye. If the robot location is on the other side of the face, it is necessary to avoid collision with the patient nose, which could be accomplished by increasing the tilt angle or by tilting the robot using a passive arm. For the current prototype, the roll mechanism assures a rotation of $360^{\circ}$ for the tool. We chose this motion range so that we could simulate many surgical procedures.
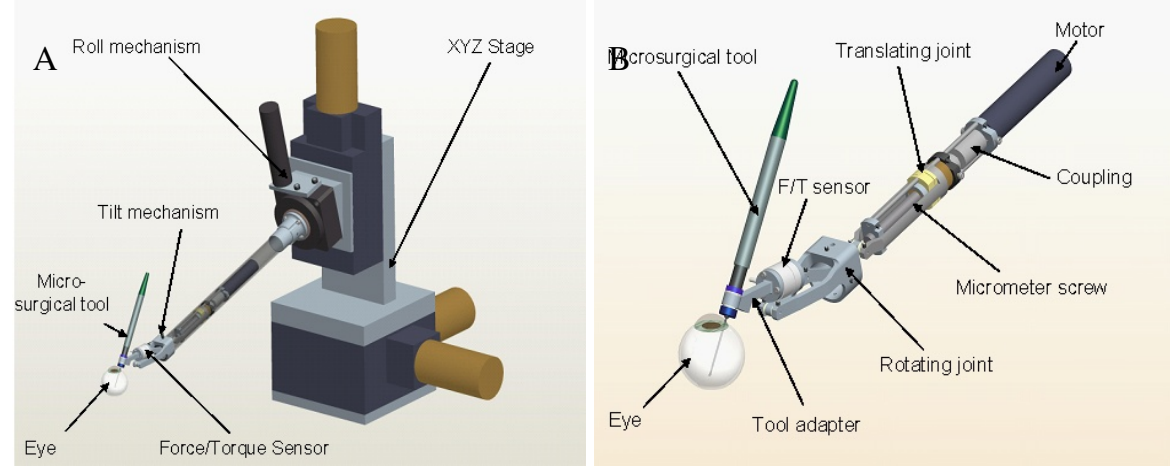

Fig. 2. Robot mechanical system (CAD model): general view $(A)$, tilt mechanism $(B)$

The tilt mechanism (Fig. 2B) is attached to the roll mechanism through a long tubular arm. In this way, nearly the entire robot is away from the surgery area. Also, this configuration assures a better possibility to separate the non-sterilized robot from the sterilized surgical area. The translating joint of the tilt mechanism is realized by a 
rotary motor and a micrometer screw without backlash. To eliminate the translating joint backlash, the slider was realized from two parts that make contact on an oblique surface. The two parts are pushed against each other by a nut through a wave spring.

The tool holder facilitates the rigid attachment of a variety of surgical instruments that are required during microsurgical procedures, such as forceps, needle holder and scissors.

The user views the operating area through a microscope and interacts with the robot by applying forces to the pipette tool holder which is attached to the robot via a force/torque sensor. Admittance control paradigm is implemented where these forces are used as input in proportional velocity control. This cooperative control of the tool provides intuitive and steady position servoing for small-scale manipulations.

\section{Experimental Methods and Results}

\subsection{Vein Cannulation Using Chorioallantoic Membrane of Chicken Embryos}

We validated the design of our robot system (Fig. 3A) by testing the ability of a user to successfully complete a vein cannulation task. We decided on a biological model; the chorioallantoic membrane (CAM) of a 12 day old chicken embryo (Fig. 3C) provides a good model for testing equipment and procedures for vitreoretinal surgery [14]. The CAM contains a large number of veins of sizes comparable to the human retina range from $40 \mu \mathrm{m}$ to $350 \mu \mathrm{m}$ [14].
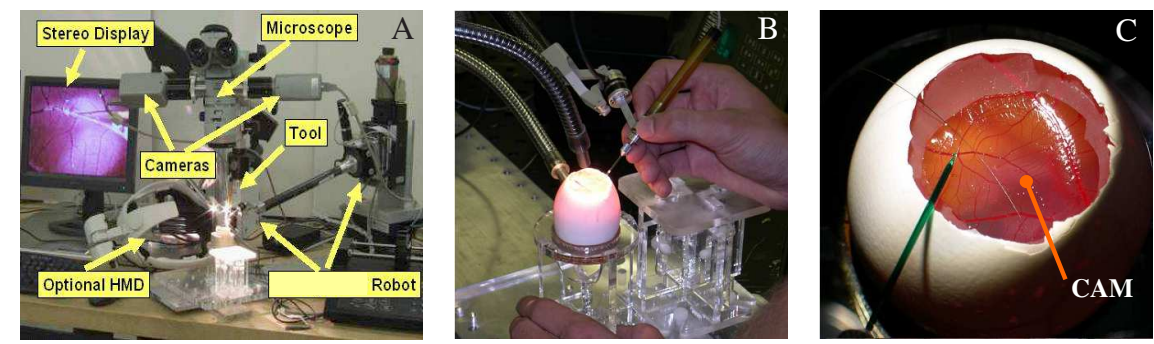

Fig. 3. (A) System Overview (B) The tool holder and micro-injection tool during a cannulation. (C) Chicken embryo CAM with a micropipette.

The following procedure was developed for reliable $\sim 80 \mu \mathrm{m}$ vein cannulation and injection of a visible marker. A pulled glass micropipette with a $1 \mu \mathrm{m}$ diameter lumen and straight tip was used for microinjection (World Precision Instruments, TIP1TW1). First, a tool designed to hold the glass micropipette was attached to the robot. A small flexible tube was run from the back of this tool to an infusion pump (PHD2000, Harvard Apparatus) filled with filtered $(0.2 \mu \mathrm{m})$ mineral oil. The pressure was set to ensure slow but steady flow of oil out of the micropipette tip. The micropipette was directly visualized under an operating stereo microscope (Zeiss OPMI MD; Carl Zeiss Surgical, Inc.). A chicken embryo was prepared as described in [14] to 
expose the CAM and placed under the operating microscope. The robot was then used to position the micropipette near the chosen CAM vein (Fig. 3B).

A study was conducted with a group of ophthalmology residents to test the ability of developing surgeons to cannulate $\sim 80 \mu \mathrm{m}$ diameter blood vessels. JHU IRB approval was obtained for this study. Subjects were asked to cannulate the blood vessel using a glass micropipette under an operating microscope. Once the tip was inside the vessel, the candidate was asked to maintain cannulation for 2 minutes. Cannulation was verified by visualizing the infusion of oil droplets into the blood column. Test subjects were divided into two groups. The Control group $(n=9)$ underwent 5 trials of cannulating the vessel freehand without the robot. The Experimental group $(n=10)$ first performed the cannulation freehand, followed by 3 trials with robot assistance, and finally a free-hand trial. At the start of the trial, the micropipette was angled to simulate its position through a sclerotomy incision during vitrectomy (Fig. 1 shows correct approach angle). The test subjects were asked to maintain, if possible, the approximate approach angle, but were not physically constrained to move in any particular way. Following parameters were measured: 1) successful cannulation; 2) time to insertion into the blood vessel; 3) maintenance of the cannulation for 2 minutes.

\subsection{Results}

Each performance was recorded using two Point Grey Flea ${ }^{\circledR}$ cameras attached to the Zeiss stereo surgical microscope. Two independent observers assessed each of the measured parameters. All free hand cannulation trials $(\mathrm{N}=64)$, including ones performed by the experimental group, were compared to the robot assisted trials $(\mathrm{N}=30)$. The following results were observed:

Table 2. Vein Cannulation using CAM of chicken embryo - Overall Performance Comparison

\begin{tabular}{|c|c|c|}
\hline Overall Results & FREEHAND $(\mathbf{N = 6 4 )}$ & ROBOT ASSISTED $(\mathbf{N}=\mathbf{3 0})$ \\
\hline Time to Cannulation (mean seconds) & 50.95 seconds & 64.88 seconds \\
Successful Cannulation & $57(89.23 \%)$ & $25(83.33 \%)$ \\
Successfully Maintained & $\mathbf{3 9}(\mathbf{6 8 . 4 2 \%})$ & $\mathbf{2 3}(\mathbf{9 2 \%})$ \\
\hline
\end{tabular}

1) For this task, there was no difference in time to cannulate a blood vessel between free-hand (51 sec) and robot assisted cannulation (64 sec; $\mathrm{p}$ value $=0.0209$ ).

2) Looking at all successful cannulations, more surgeons using the robot maintained the micropipette tip within the blood vessel for the requested 2 minutes $(68.4 \%$ (free-hand) to $92 \%$ (robot assisted); $\mathrm{p}$ value $=0.00298$ ).

3) There was significant improvement with repeated attempts when using the robot (Table 3). While the first two robot trials averaged 82 and $83 \mathrm{sec}$ to cannulation, the same subjects reduced cannulation time to $27 \mathrm{sec}$ on the third trial ( $\mathrm{p}$ value $=0.0119$ ). Some improvement was noticed in the free-hand cannulation attempts, although not statistically significant. Mean time in trial 5 for both groups increased significantly, this could be attributed to fatigue and in the case of the experimental group difficulty in readjusting to freehand mode. 
Table 3. Vein Cannulation using CAM of chicken embryo - Study results

\begin{tabular}{|c|c|c|c|c|c|c|}
\hline & \multicolumn{3}{|c|}{ CONTROL } & \multicolumn{3}{|c|}{ EXPERIMENTAL* } \\
\hline & \multicolumn{2}{|c|}{$\begin{array}{c}\text { Time to Cannula- } \\
\text { tion (sec) }\end{array}$} & $\begin{array}{l}\text { Successfully } \\
\text { Maintained }\end{array}$ & \multicolumn{2}{|c|}{$\begin{array}{c}\text { Time to Cannula- } \\
\text { tion (sec) }\end{array}$} & $\begin{array}{l}\text { Successfully } \\
\text { Maintained }\end{array}$ \\
\hline & MEAN & STDEV & & MEAN & STDEV & \\
\hline 1st Trial & 89.50 & 72.567 & $75.00 \%$ & 51.50 & 36.726 & $75.00 \%$ \\
\hline 2nd Trial & 27.25 & 20.617 & $50.00 \%$ & 82.14 & 63.734 & $85.71 \%$ \\
\hline 3rd Trial & 21.14 & 11.894 & $57.14 \%$ & 83.20 & 100.09 & $90.00 \%$ \\
\hline 4th Trial & 26.25 & 23.015 & $75.00 \%$ & 26.88 & 29.434 & $100.00 \%$ \\
\hline 5th Trial & 49.00 & 74.057 & $88.88 \%$ & 84.33 & 83.833 & $55.55 \%$ \\
\hline
\end{tabular}

*Note: Trials 2, 3, 4 in Experimental group are robot assisted (shaded). Trials 1 and 5 are free-hand.

We concluded that with some learning, the robot improved the surgical performance of maintaining the cannulation, and after two trials, improved the ability to cannulate the blood vessel. The geometry of the experimental set up allowed the surgeon plenty of latitude to position his/her hand and pipette in a comfortable position. Due to the straight tip of the pipette the subjects naturally navigated towards more horizontal approach (Fig. 2C) to the surface of the CAM, which does not adequately simulate vitreoretinal surgery (Fig 1). We believe that if the surgeons were restricted in hand placement or the tool was constrained to better simulate vitreoretinal surgery, the differences between the free-hand and robot assisted results would have been greater.
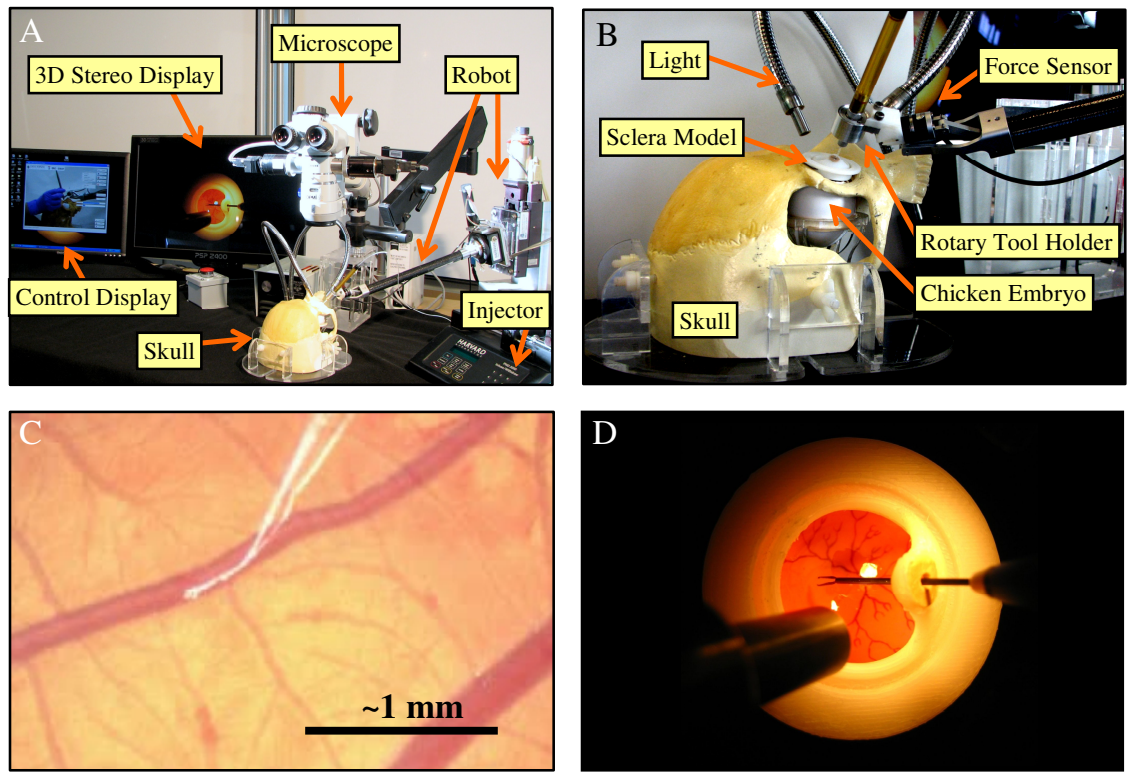

Fig. 4. (A) Updated testbed (B) Close-up of the simulated operating area. (C) Bent tip pipette during cannulation (D) Sclera model with constrained instrument entry point and grasper tool. 


\section{Current and Future Work}

The above experiment demonstrated that robot assisted micro-vessel injection is feasible and shows potential in vitreoretinal surgery. Pursuing this idea further we have build a more realistic operating environment. It includes a Sawbones@ scull with a sclera phantom placed in the eye socket. The chicken embryo is placed inside the skull directly under the eye socket such that the chorioallantoic membrane is $\sim 23 \mathrm{~mm}$ from the sclera's top surface. The sclera model consists of a 3D rapid prototyped ring with a rubber gasket in which a $2 \mathrm{~mm}$ incision was made (Fig. 4D). This mimics a typical sclerotomy port, where the instrument is constrained to insertion and pivoting about the semi elastic entry point.

The robot was also outfitted with a new tool holder that allows free rotation of the instrument about tool's main axis (Fig 4B). This adds an additional passive degree of freedom to the robot, a desired feature for use with instruments that are not axisymmetric, e.g. bent tip pipette.

\subsection{Improved Vein Cannulation Experiment}

The initial experiments performed with a straight tip pipette proved to be not compatible with the geometric constraints imposed by the operating environment during retinal vein cannulation. In order to orient the pipette tangentially to the vessel and respect the entry point (Fig. 1), a number of bent tip micropipettes were considered. Initially, thin ( 10mm long) neck bent tip pipettes (Eppendorf, \#930-00-103-1) with 4um ID and 7um OD were tested. However, these were too flexible under the forces required to puncture the CAM vessels, and were also difficult to localize under the microscope. A short neck bent tip pipette was successfully employed for cannulation (MIC-50-45, Humagen Fertility Diagnostics, Inc.). The pipette is bent at 45 degrees, $0.5 \mathrm{~mm}$ from the tip, and has a $5 \mu \mathrm{m}$ ID, a beveled tip and a spike. This type of pipette provided the necessary rigidity, sufficient visual localization, and the geometry required for tangential approach to the vessel and a sharp tip for easy vessel wall puncture (Fig. 4C). The aforementioned experiment with Humagen micropipette and the new accurate testbed is ongoing. The next step is to migrate to an in vivo model (rabbit retina).

\section{Conclusions}

We have developed a cooperative robot for retinal microsurgery which, along with accompanying phantoms, surgical instruments and visualization equipment, is a reliable and replicable testbed for developing and evaluating various computer assisted eye surgery procedures and new instruments.

The CAM vein cannulation experiments showed feasibility of using the robot to successfully cannulate veins down to $\sim 80 \mu \mathrm{m}$ rapidly, reliably, and with minimal damage to the surrounding tissue. The results of the cannulation comparison study suggest that robot assistance improves performance in maintaining the micropipette inside the cannulated vein and with slight learning curve improves the time to cannulation. However, the experimental setup was limited in terms of accurately simulating 
real surgical operating conditions. This is addressed in the improvements to the robot interface with standard surgical instruments, to the operating phantom in adding realistic geometric constraints and also system enhancements, such as the 3D stereo LCD display. The next set of experiments will formally test the feasibility of cannulation in the new, improved environment, in which, we hypothesize, the surgeons will find cannulating freehand more difficult then with the robot assistance. Encouraged by our experience presented here we are convinced that we can employ our system in other challenging ophthalmological procedures such as internal limiting membrane peeling.

\section{References}

1. World Health Organization News Release (2004), http: //www. who.int/mediacentre/news/releases

2. Patkin, M.: Ergonomics applied to the practice of microsurgery. Aust. NZ J. Surg. 47, 320-329 (1977)

3. Gupta, P., Jensen, P., de Juan, E.: Surgical forces and tactile perception during retinal micro-surgery. MICCAI, 1218-1225 (1999)

4. Sinch, S., Riviere, C.: Physiological tremor amplitude during retinal microsurgery. In: IEEE Northeast Bioengineering Conference, pp. 171-172 (2002)

5. Taylor, R.H., Stoianovici, D.: Medical Robotics in Computer-Integrated Surgery. IEEE Transactions on Robotics and Automation 19, 765-781 (2003)

6. Chui, C.K., Hguyen, H.T., Wang, Y., Mullick, R., Raghavan, R., Anderson, J.: Potential field and anatomy vasculature for real time computation in daVinci. In: First Visible Human Conference, Bethesda, USA (1996)

7. Ikuta, K., Yamamoto, K., Sasaki, K.: Development of remote microsurgery robot and new surgical procedure for deep and narrow space. In: IEEE ICRA, pp. 1103-1108 (2003)

8. Charles, S., Williams, R.E., Hamel, B.: Design of a Surgeon-Machine Interface for Teleoperated Microsurgery. IEEE Engineering in Medicine and Biology Society, 883-884 (1989)

9. Hunter, I.W., Doukoglou, D., Lafontaine, S.R., Charette, G., Jones, L.A., Sagar, M.A., Mallison, G.D., Hunter, P.J.: A teleoperated microsurgical robot and associated virtual environment for eye surgery. Presence 2, 265-280 (1993)

10. Kumar, R., Hager, G.D., Barnes, A., Jensen, P., Whitcomb, L.L., Taylor, R.H.: An augmentation system for fine manipulation. In: Delp, S.L., DiGoia, A.M., Jaramaz, B. (eds.) MICCAI 2000. LNCS, vol. 1935, pp. 956-965. Springer, Heidelberg (2000)

11. Taylor, R.H., Jensen, P., Whitcomb, L.L., Barnes, A., Kumar, R., Stoianovici, D., Gupta, P., Wang, Z., de Juan, E., Kavoussi, L.: Steady-hand robotic system for microsurgical augmentation. The International Journal of Robotics Research 18(12), 1201-1210 (1999)

12. Iordachita, I., Kapoor, A., Mitchell, B., Kazanzides, P., Hager, G., Handa, J., Taylor, R.: Steady-Hand Manipulator for Retinal Surgery. In: MICCAI, Medical Robotics Workshop (2006)

13. Mitchell, B., Koo, J., Iordachita, I., Kazanzides, P., Kapoor, A., Handa, J., Hager, G., Taylor, R.: Development and Application of a New Steady-Hand Manipulator for Retinal Surgery. In: IEEE ICRA, pp. 623-629 (2007)

14. Leng, T., Miller, J., Bilbao, K., Palanker, D., Huie, P., Blumenkranz, M.: The chick chorioallantoic membrane as a model tissue for surgical retinal research and simulation. Retina 24(3), 427-434 (2004) 